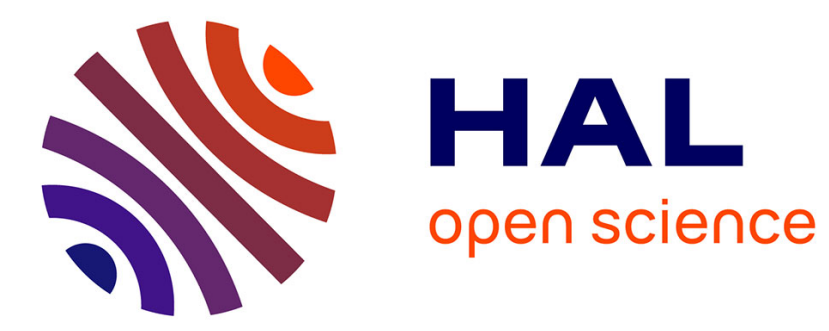

\title{
Evidence of Subproton-Scale Magnetic Holes in the Venusian Magnetosheath
}

Katherine A Goodrich, John W Bonnell, Shannon Curry, Roberto Livi, Phyllis Whittlesey, Forrest Mozer, David Malaspina, Jasper Halekas, Michael Mcmanus, Stuart Bale, et al.

\section{To cite this version:}

Katherine A Goodrich, John W Bonnell, Shannon Curry, Roberto Livi, Phyllis Whittlesey, et al.. Evidence of Subproton-Scale Magnetic Holes in the Venusian Magnetosheath. Geophysical Research Letters, 2021, 48 (5), 10.1029/2020GL090329 . insu-03176048

\section{HAL Id: insu-03176048 https://hal-insu.archives-ouvertes.fr/insu-03176048}

Submitted on 22 Mar 2021

HAL is a multi-disciplinary open access archive for the deposit and dissemination of scientific research documents, whether they are published or not. The documents may come from teaching and research institutions in France or abroad, or from public or private research centers.
L'archive ouverte pluridisciplinaire HAL, est destinée au dépôt et à la diffusion de documents scientifiques de niveau recherche, publiés ou non, émanant des établissements d'enseignement et de recherche français ou étrangers, des laboratoires publics ou privés. 


\section{Geophysical Research Letters}

\section{RESEARCH LETTER \\ 10.1029/2020GL090329}

Special Section:

Parker Solar Probe Observa-

tions at Venus: VGA1-2

\section{Key Points:}

- Magnetic depressions with spatial scales less than the local proton gyroradius are observed in the Venusian magnetosheath

- The amplitude and features of the electric field associated with these structures are consistent with electron vortices, though they deviate in direction by $90^{\circ}$

- Similar structures have been observed in the terrestrial magnetosphere, suggesting they are part of a universal plasma process

Correspondence to:

K. A. Goodrich,

katygoodrich@berkeley.edu

Citation:

Goodrich, K. A., Bonnell, J. W., Curry, S., Livi, R., Whittlesey, P., Mozer, F., et al. (2021). Evidence of subprotonscale magnetic holes in the Venusian magnetosheath. Geophysical Research Letters, 48, e2020GL090329. https://doi. org/10.1029/2020GL090329

Received 18 AUG 2020 Accepted 5 JAN 2021

(C) 2021. American Geophysical Union. All Rights Reserved.

\section{Evidence of Subproton-Scale Magnetic Holes in the Venusian Magnetosheath}

\author{
Katherine A. Goodrich ${ }^{1}$ (D) John W. Bonnell ${ }^{1}$, Shannon Curry ${ }^{1}$ (D), Roberto Livi ${ }^{1}$ (D), \\ Phyllis Whittlesey $^{1}$, Forrest Mozer ${ }^{1,2}$ (D), David Malaspina ${ }^{3}$ (D), Jasper Halekas ${ }^{4}$ (D), \\ Michael McManus ${ }^{1,2}$, Stuart Bale ${ }^{1,2}$, Trevor Bowen ${ }^{1}$ (D), Anthony Case ${ }^{5}$ (D), \\ Thierry Dudok de Wit ${ }^{6}$ (D), Keith Goetz ${ }^{7}$ (D), Peter Harvey ${ }^{1}$, Justin Kasper ${ }^{8}$, Davin Larson ${ }^{1}$, \\ Robert MacDowall ${ }^{9}$ (D), Marc Pulupa ${ }^{1}$ (D), and Michael Stevens 5 (D)
}

${ }^{1}$ Space Sciences Laboratory, University of California, Berkeley, CA, USA, ${ }^{2}$ Department of Physics, University of California, Berkeley, CA, USA, ${ }^{3}$ Department of Astrophysical and Planetary Sciences, University of Colorado Boulder, Boulder, CO, USA, ${ }^{4}$ Department of Physics and Astronomy, University of Iowa, Iowa City, IA, USA, ${ }^{5}$ HarvardSmithsonian Center for Astrophysics, Cambridge, MA, USA, ${ }^{6}$ Laboratoire de Physique et Chimie de l'Environnement et de l'Espace (LPC2E/CNRS), University of Orléans, Orléans, France, ${ }^{7}$ School of Physics and Astronomy, University of Minnesota, Minneapolis, MN, USA, ${ }^{8}$ Climate and Space Sciences and Engineering, University of Michigan, Ann Arbor, MI, USA, ${ }^{9}$ NASA, Goddard Space Flight Center, Greenbelt, MD, USA

Abstract Depressions in magnetic field strength, commonly referred to as magnetic holes, are observed ubiquitously in space plasmas. Subproton-scale magnetic holes with spatial scales smaller than or on the order of a proton gyroradius, are likely supported by electron current vortices, rotating perpendicular to the ambient magnetic field. While there are numerous accounts of subproton-scale magnetic holes within the Earth's magnetosphere, there are few, if any, reported observations in other space plasma environments. We present the first evidence of subproton-scale magnetic holes in the Venusian magnetosheath. During Parker Solar Probe's first Venus Gravity Assist, the spacecraft crossed the planet's bow shock and subsequently observed the Venusian magnetosheath. The FIELDS instrument suite onboard the spacecraft achieved magnetic and electric field measurements of magnetic hole structures. The electric fields associated with magnetic depressions are consistent with electron current vortices with amplitudes on the order of $1 \mu \mathrm{A} / \mathrm{m}^{2}$.

Plain Language Summary The Sun is constantly ejecting an ionized gas, or plasma. This plasma from the Sun is called the solar wind and usually consists of an equal number of negatively charged electrons and their larger positively charged counterparts, protons. These particles travel together from the Sun, canceling out each other's charge. When the plasma encounters obstacles, however, like the Earth or Venus, the plasma becomes disturbed. This can cause the electrons to separate from the protons and form unbalanced structures. One interesting structure that has recently been discovered at Earth are electron vortices. These vortices can create their own magnetic and electric fields and slightly alter the plasma around them. We have seen electron vortices where the solar wind meets the Earth, but are not sure how they are created or how strongly they affect the plasma around them. We report, for the first time, evidence of electron vortices where the solar wind encounters Venus. These new findings show the process that creates electron vortices takes place at both Earth and Venus, strongly implying a universal process in space.

\section{Introduction}

Subproton-scale magnetic holes are depressions in total magnetic field (B) strength with spatial scales less than, or on the order of, a proton gyroradius $\left(\rho_{p}\right)$. Depressions in $|\mathbf{B}|$ that are spatially larger than a proton gyroradius $\left(\rho_{p}\right)$ can usually be attributed to the magnetic mirror instability (Southwood \& Kivelson, 1993), so much so they are commonly referred to as "mirror mode waves." Mirror mode waves have been observed frequently in multiple space plasma environments such as the solar wind (Russell et al., 2008; Wintertialter et al., 1994) and terrestrial magnetosheath (Johnson \& Cheng, 1997; Soucek et al., 2008). They are generally known to be generated via a plasma temperature anisotropy (Califano et al., 2008; Hasegawa, 1969; Kuznetsov et al., 2008). 
Unlike mirror waves, subproton-scale magnetic holes are observed with features consistent with current layers carried by electrons (Gershman et al., 2016; Goodrich et al., 2016a). While the structure may extend longer than a $\rho_{p}$ (Goodrich et al., 2016a), the current layers associated with subproton-scale magnetic holes have spatial scales smaller than $\rho_{p}$. Subproton-scale magnetic holes have been observed within the Earth's magnetosphere during times of magnetic field fluctuations, particularly in the magnetosheath (Huang et al., 2017; H. Liu et al., 2019; Yao et al., 2017) and near-Earth plasma sheet (Ge et al., 2011; Gershman et al., 2016; Sun et al., 2012; Sundberg et al., 2015; Tenerani et al., 2012, 2013). Currents carried by such electron vortices have been observed both through high resolution particle measurements from the Magnetospheric Multiscale (MMS) Mission (Gershman et al., 2016) as well as electric field measurements (Goodrich et al., 2016b) from both MMS and THEMIS (Goodrich et al., 2016a).

The generation of subproton-scale magnetic holes are an open question. Some have suggested they are a variation of electron solitary waves (Ji et al., 2014; Li et al., 2016). They are also thought to arise through a the nonlinear evolution the mirror instability and the tearing instability (Ahmadi et al., 2017; Balikhin et al., 2010, 2012). Additionally, the simulations performed by Haynes et al. (2015) and Roytershteyn et al. (2015) suggest subproton-scale magnetic holes arise as a coherent structure in plasma turbulence. None of these theories have been observationally confirmed.

While observations of subproton-scale magnetic holes have become increasingly frequent in recent years, their role and importance to space plasma physics is not well known. Confirmed reports of subproton-scale magnetic holes in both the terrestrial magnetosheath and plasma sheet suggest they may be a product of a universal process. They have even been shown to energize electrons as they shrink to smaller spatial sizes (H. Liu et al., 2019; J. Liu et al., 2020). However, there are currently no observations of such signatures that extend beyond the terrestrial magnetosphere, though a possible candidate has been identified in the solar wind (Y. Y. Liu et al., 2020). This is likely due to the fact that structures with this spatial scale are difficult to observe given the time resolution limitations on particle instruments available on previous missions to Venus, Mercury, and Mars. Additionally, the majority of these missions do not possess a full range of electric field observations, which can also be used to observe electron currents.

We report, for the first time, evidence of structures bearing significant similarities to subproton-scale magnetic holes in the Venusian magnetosheath. These structures were observed by the Parker Solar Probe (PSP) spacecraft during its initial Venus Gravity Assist (VGA1). Significant depressions in magnetic field strength (up to $30 \%$ of the original $|\mathbf{B}|$ value) were observed at length scales less than the local thermal proton gyroradius throughout the Venusian magnetosheath. These magnetic depressions have corresponding unipolar and bipolar electric field signals that are consistent with the presence of electron vortices. The amplitude of these electric fields is additionally consistent with the presence of a $1.75 \mu \mathrm{A} / \mathrm{m}^{2}$ current vortex. The direction of the electric field, however, deviates $90^{\circ}$ from its expected value.

In this paper, we review the observations from VGA1, and the magnetic hole structures found within. We then compare these observations with a simple model of an electron vortex. This comparison shows the observed signatures are largely consistent with electron vortices. These observations bear strong similarities to subproton-scale magnetic holes observed in the terrestrial magnetosphere. This report suggests these structures are indicative of a universal, or pervasive, process in magnetospheric plasmas.

\section{Data and Instruments}

The measurements examined in this study are taken from the Parker Solar Probe mission (Fox et al., 2016). Its purpose is to measure the "young" solar wind by obtaining measurements as close as nine solar radii from the surface of the Sun. In order for the spacecraft to reach this destination, it must encounter Venus seven times for gravitational assistance. Here we examine fields and particle measurements taken during the first Venus gravity assist, heretofore referred to as VGA1, on October 3, 2018 between 07:00 and 08:50 UTC.

Observations of electric field and magnetic field were obtained via the FIELDS instrument suite (Bale et al., 2016; Malaspina et al., 2016). This suite measures magnetic field from two fluxgate magnetometers (FGM) as well as a search coil magnetometer (SCM), all of which are mounted on the magnetometer boom 


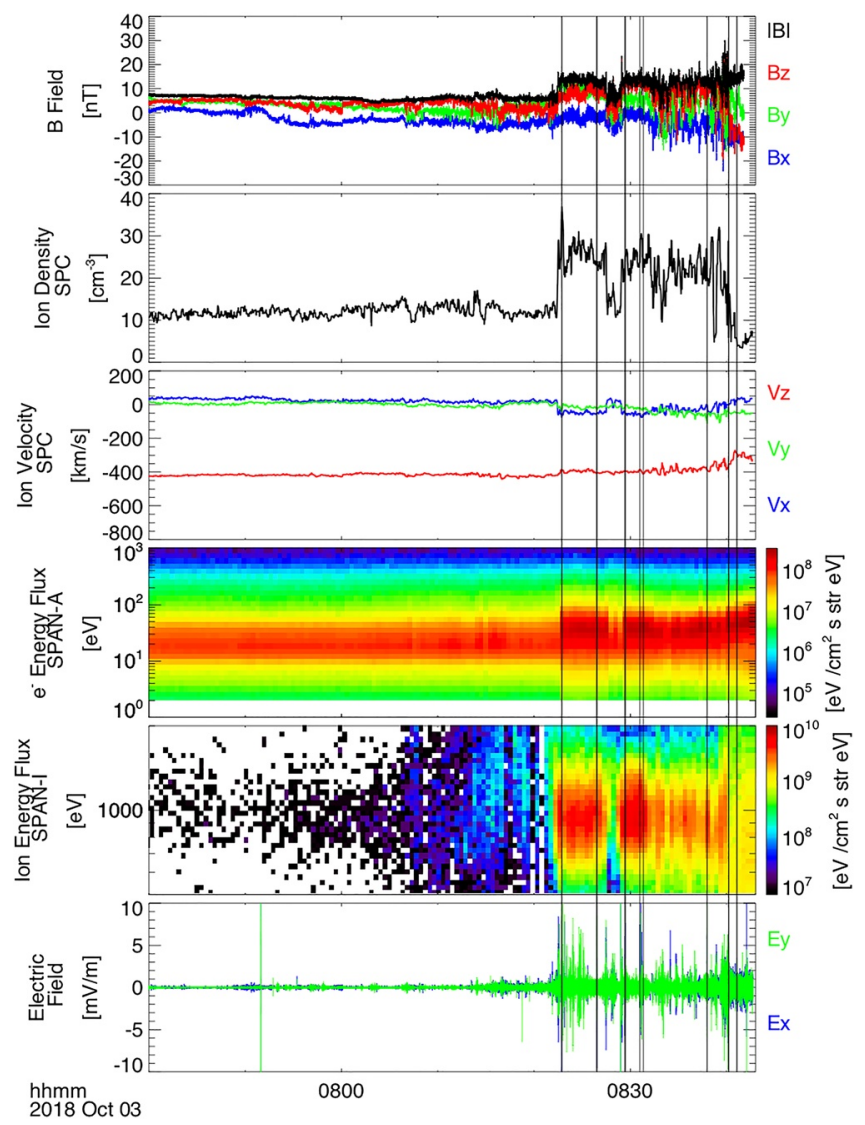

Figure 1. Overview of the first Venus Flyby undertaken by Parker Solar Probe. The plot shows, in descending order, magnetic field, proton density from Solar Probe Cup (SPC), proton velocity from SPC, electron energy flux, proton energy flux from SPAN-ion, and electric field. All vectors are in spacecraft coordinates. The Parker spacecraft initially measured solar wind before encountering the Venusian shock at 08:22:20 UTC. It then observed the Venusian magnetosheath as well as other bow shock crossings before the end of the encounter at 08:50. All vertical lines mark times in which subproton-scale magnetic holes were observed. directly behind the heat shield. Four $2 \mathrm{~m}$ antennas, which measure electric potentials $V_{1}, V_{2}, V_{3}$, and $V_{4}$ are positioned in the plane of the heat shield, perpendicular to the sun-spacecraft direction. The fifth potential, $V_{5}$, is measured by a $21 \mathrm{~cm}$ antenna, also mounted on the magnetometer boom. The electric field in the plane of the heat shield is derived from the differential voltage measurements $\left(V_{1}-V_{2}\right.$ and $\left.V_{3}-V_{4}\right)$ calculated on the spacecraft.

The electric fields were calibrated by least squares fitting 12 second averages of $E_{X}$ versus $-\left(v_{i} \times \mathbf{B}\right)_{X}$ and $E_{Y}$ versus $-\left(v_{i} \times \mathbf{B}\right)_{Y}$, where $v_{i}$ is the proton velocity from the Solar Probe Cup (SPC) (Case et al., 2020). The four least squares coefficients were two dc offsets resulting from electronic offsets, the effective antenna length, and an angular rotation of the fields in the X-Y plane. This rotation was found necessary and may have resulted because the electric field antenna was comparable in size to the spacecraft and the Debye length, as described further in Mozer et al. (2020).

All particle measurements used in this analysis were provided by the Solar Wind Electrons Alphas and Protons (SWEAP) instrument suite (Kasper et al., 2016). Electron moments and distributions were measured by the SPAN-electron instrument (Halekas et al., 2020; Whittlesey et al., 2020). Ion moments and distributions were measured by SPC and SPAN-ion (Kasper et al., 2016) instruments. SPC has a $40^{\circ}$ half-angle field of view, with its center pointed directly sunward. SPAN-ion has a $120^{\circ} \times 247.5^{\circ}$ view of the sky perpendicular to the sunward direction. The combination of SPC and SPAN-ion provides a nearly full view of the sky. During VGA1, SPC had a $1.3 \mathrm{~s}$ temporal resolution. SPAN-electron and SPAN-ion had a temporal cadence of $\sim 28 \mathrm{~s}$.

Figure 1 shows an overview of VGA1, which displays magnetic field, proton density $\left(n_{p}\right)$, proton velocity $\left(\mathbf{V}_{\mathbf{p}}\right)$, electron energy flux, ion energy flux from SPAN-ion, and high pass filtered electric field (all signal below $1 \mathrm{~Hz}$ removed), in descending order. All vectors are shown in the spacecraft frame, where $Z$ is pointed sunward and $X$ is pointed along the spacecraft trajectory in the plane of the heat shield. It is of note that these measurements are the first ever current-biased DC electric field measurements at Venus.

All proton measurements examined are taken from SPC unless otherwise stated. For all $\mathbf{V}_{\mathbf{p}}, n_{p}$, and temperature ( $T_{p}$, not displayed) moments, the times at which $n_{p}=0$ were removed. All data were subsequently median smoothed over 11 consecutive point intervals. The focus of this study are structures with spatial scales less than $\rho_{p}$, which are observed over tens of milliseconds. This time frame is well below the time resolution of all available particle instruments and therefore this treatment of the particle data is appropriate to provide overall context of the plasma environment during VGA1.

The PSP spacecraft made its approach traveling in the sunward direction and encountered the Venusian environment on its dawnward-flank side. Between 07:00 and 08:00 UTC, the spacecraft detected solar wind plasma. This is evident from steady proton density and antisunward velocity at $10 \mathrm{~cm}^{-3}$ and $450 \mathrm{~km} / \mathrm{s} \mathrm{re-}$ spectively. There are no coherent features observed by SPAN-ion and the magnetic field remains at a constant amplitude of $\sim 5 \mathrm{nT}$. The spacecraft subsequently (between 08:00 and 08:22 UTC) observes magnetic fluctuations and broad energy signals in ion energy flux from SPAN-ion. This indicates ion flows outside of the SPC field of view, which is consistent with the presence of reflected ions from the Venusian bow shock.

PSP likely crossed the Venusian bow shock and entered the magnetosheath for the first time at 08:22:20 UTC. This is indicated by the abrupt increase in $|\mathbf{B}|$ and $n_{p}$, as well as a deviation in proton velocity. The spacecraft subsequently crossed the bow shock approximately 5 times before it approached the magnetic pile-up 


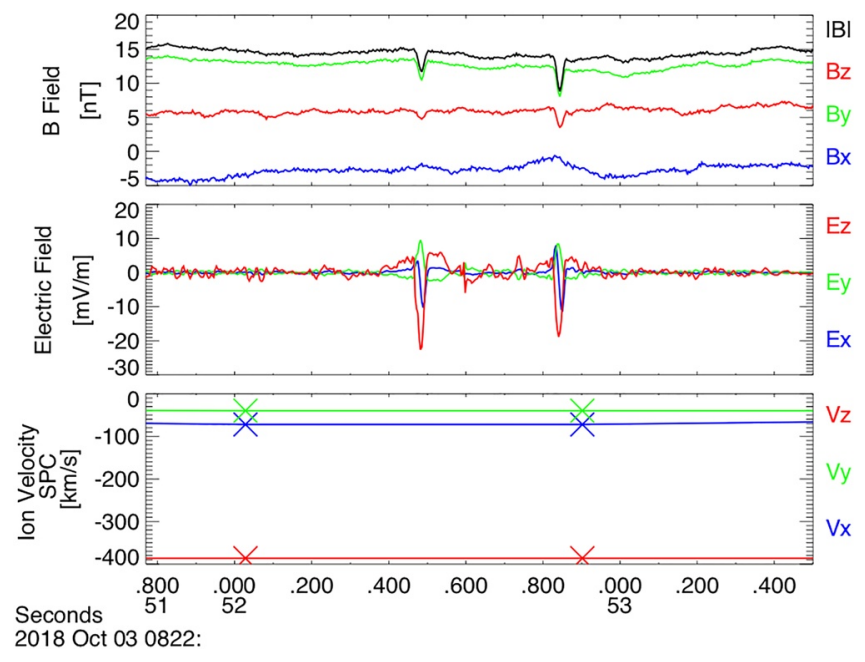

Figure 2. Two example magnetic hole candidates. This figure shows a $1.65 \mathrm{~s}$ zoomed in view of the magnetic field, electric field, and proton velocity at 08:22:52 UTC, approximately $30 \mathrm{~s}$ after Parker Solar Probe made its initial Venusian bow shock crossing. Bipolar and unipolar electric field signatures are observed in tandem with localized (50 ms) depressions in magnetic field strength. region at 08:50 UTC. At this time all instruments were powered off due to a solar limb sensor anomaly, and no further data were collected during the encounter.

The vertical lines in Figure 1 highlight times in which subproton-scale magnetic hole candidates were observed. Eleven candidates were identified after the initial bow shock crossing in the Venusian magnetosheath. These structures were identified by a distinct decrease in $|\mathbf{B}|$, as well as corresponding $\mathbf{E}$ field signatures, with observation times over tens of milliseconds. The candidates identified showed no overall change in the average (over $1 \mathrm{~s}$ ) magnetic field. They were also observed alongside electric field signatures that will be discussed in depth in the following sections of this paper. All candidates were found within the Venusian magnetosheath. No magnetic holes were observed in the solar wind or foreshock regions prior to observing the initial shock crossing, suggesting they are generated through a process that takes place within the Venusian magnetosheath.

\section{Magnetic Hole Observations}

Figure 2 shows an example of two magnetic hole candidates. It shows a $1.65 \mathrm{~s}$ zoomed in view of the magnetic field, electric field and proton velocity at $\sim 08: 22: 52$ UTC, $\sim 30 \mathrm{~s}$ after the spacecraft's initial encounter with the Venusian bow shock. All vectors are shown in the spacecraft frame. $E_{x}$ and $E_{y}$ are directly measured by the four voltage probes in the plane aligned with the heat shield. $E_{z}$ is calculated under the assumption that $\mathbf{E} \cdot \mathbf{B}=0$. This assumption is appropriate as all observed electric field associated with subproton-scale magnetic holes have been primarily perpendicular to the magnetic field (Goodrich et al., 2016b).

The observed $\Delta|\mathbf{B}| /|\mathbf{B}|$ for each event is $\sim 35 \%(\sim 5 / 14 \mathrm{nT})$ and the magnetic field direction shows little deviation $\left(\sim 2^{\circ}\right)$ from the surrounding magnetic field. Both events are observed over $50 \mathrm{~ms}$. The spatial length of the structure can be found under the assumption that it is stationary in the plasma (i.e., proton) frame. Subproton-scale magnetic holes have also been shown to travel with the plasma by H. Liu et al. (2019). The spacecraft speed at this time is $5 \%$ of the measured proton speed and considered insignificant in this calculation. The spatial length of the magnetic holes are estimated to be $20 \mathrm{~km}$, as the protons are measured to travel $\sim 400 \mathrm{~km} / \mathrm{s}$ antisunward. This scale falls within the subproton-scale as the estimated proton gyroradius in this region is $40 \mathrm{~km}\left(\sqrt{m_{p} T_{p} / \mathbf{B}^{2}}\right.$, derived via observations from the flux gate magnetometer and proton temperature moments from SPC). These characteristics are all consistent with prior observations of subproton-scale magnetic holes in the terrestrial context.

Electric field signals are seen in conjunction with the observed magnetic field depressions. A unipolar pulse reaching $\sim 10$ and $\sim 20 \mathrm{mV} / \mathrm{m}$ is seen in the $Y$ and $Z$ directions respectively. A bipolar signal with an amplitude of $\sim 10 \mathrm{mV} / \mathrm{m}$ is seen in the $X$ direction. These signatures are qualitatively consistent with subproton-scale magnetic holes observed in the Earth's magnetosphere.

\section{Model}

In order to interpret these observations, we propose of a model of a subproton-scale magnetic hole and compare it's magnetic and electric field structures to the observed features. We construct a cylindrically symmetric current vortex. The current in this model is carried solely by electrons and is stationary in the plasma frame. The current $J_{\phi}$ is defined as

$$
J_{\phi}=\left\{\begin{array}{cc}
J_{0} \sin \left(\frac{\pi r}{2 R}\right) & \text { if } \mathrm{r} \leq \mathrm{R} \\
0 & \text { if } \mathrm{r}>\mathrm{R}
\end{array}\right.
$$


where $r$ is the radial distance from the center of the magnetic hole and $R$ is the estimated radius of the magnetic hole structure. $J_{0}$ is the maximum current density within the structure. We then simulated a spacecraft crossing this structure in various trajectories.

Multiple trajectories and values of $J_{0}$ and $R$ were tested with this model. All trajectories were parallel to the along-track direction, while the offset distance from the center of the structure in the cross-track direction varied. The trajectory is assumed to be perpendicular to the axis of symmetry of the vortex. The magnetic and electric field induced by the vortex were then calculated based on the defined spacecraft trajectory.

The induced magnetic field from this current is derived using Ampere's law,

$$
\Delta \mathbf{B}_{\mathbf{Z}}\left(r_{S C}\right)=\frac{\mu_{0}}{R} \int_{r_{S C}}^{R} J_{\phi}(r) r d r
$$

The resulting magnetic field then becomes $\mathbf{B}_{\mathrm{Z}}\left(r_{\mathrm{SC}}\right)=\mathbf{B}_{\mathrm{Z}}(R)-\Delta \mathbf{B}_{\mathrm{Z}}\left(r_{\mathrm{SC}}\right)$, where $r_{\mathrm{SC}}$ is the radial position of the simulated spacecraft. The electric field was derived via the Lorentz equation (Stix, 1992),

$$
\mathbf{E}_{\mathbf{R}}\left(x_{S C}, y_{S C}\right)=-\mathbf{v}_{\mathbf{e}}\left(x_{S C}, y_{S C}\right) \times \mathbf{B}_{\mathbf{Z}}\left(r_{S C}\right) .
$$

The electron velocity as a function of spacecraft position, $\mathbf{v}_{\mathbf{e}}\left(x_{\mathrm{SC}}, y_{\mathrm{SC}}\right)$, was determined by $\mathbf{v}_{\mathbf{e}}=-J_{\phi}\left(r_{\mathrm{SC}}\right) / q n_{e}$. $\mathbf{v}_{\mathbf{e}}$ is estimated to be on the order of $2,000 \mathrm{~km} / \mathrm{s}$, calculated from $\mathbf{E} \times \mathbf{B}$ measurements from PSP. The density of the current layer, $n_{e}$, can therefore be estimated by $J_{0} / q \mathbf{E}_{\mathbf{R}} \times \mathbf{B}_{\mathbf{Z}}$. It should also be noted that the electric field used here was used only to make initial estimates of the model. The model is mainly constrained to the observed magnetic field.

The parameters of the model, particularly the radius of the structure $(R)$, current density amplitude $\left(J_{0}\right)$, and offset of the trajectory from the center of the vortex were all varied to best replicate the characteristics of the second magnetic hole candidate in Figure 2. Under the assumption that the structure is stationary in the plasma frame $R$ must be on the order of $V_{\mathrm{SPC}} \Delta t / 2(10 \mathrm{~km}) . J_{0}$ was chosen such that the induced magnetic field produced the same $\Delta|\mathbf{B}|$ observed by PSP $(\sim 5 \mathrm{nT})$.

We found the following values to be consistent with the chosen example:

- $R=15 \mathrm{~km}$

- $J_{0}=1.75 \mu \mathrm{A} / \mathrm{m}^{2}$

- Offset $=9 \mathrm{~km}$

- $n_{e}=5.5 \mathrm{~cm}^{-3}$

Figure 3 shows a direct comparison between the observed magnetic and electric field of the subproton-scale magnetic hole ( $b$ and d) and those derived by the model ( $a$ and $c$ ) with the listed parameters. The observed $\mathbf{E}_{\mathbf{R}}$ and $\mathbf{B}_{\mathbf{Z}}$ vectors in this figure were rotated such that the red vector ("B") is aligned with the magnetic field. The blue vector ("along") signifies the proton flow direction (perpendicular to the magnetic field), this is analogous to the "along-track" direction. The green vector ("cross") is aligned in the "cross-track" direction. This can be considered the "plasma frame" as the deviation in electric field under the Lorentz transformation is negligible ( $4 \%$ of the observed value).

The modeled magnetic field decreases by $5.3 \mathrm{nT}$, matching the observed $\Delta|\mathbf{B}|$ observed by PSP (5.2 nT). This overall decrease is observed over $23 \mathrm{~km}$ in the model, which is further consistent with the observation time of the structure $(\sim 20 \mathrm{~km})$. The modeled electric fields also bear certain similarities to observations. First, the amplitudes of the modeled electric field $(\sim 9.75$ and $16 \mathrm{mV} / \mathrm{m}$ for along and cross track respectively) are consistent with those observed ( $\sim 25$ and $\sim 8 \mathrm{mV} / \mathrm{m}$ ). The ratio of these amplitudes is approximately $1 / 2$ in the model and $1 / 3$ in observations, suggesting the modeled trajectory offset is consistent with the trajectory of the PSP spacecraft.

The electric fields derived from the model, however, deviate in direction from the observations by $\sim 90^{\circ}$. It is unclear, at this time, what the reason is for this deviation. One source of error may be that the full plasma flow in the Venusian magnetosheath may lie partially outside of the field of view of the SPC and SPAN-ion 

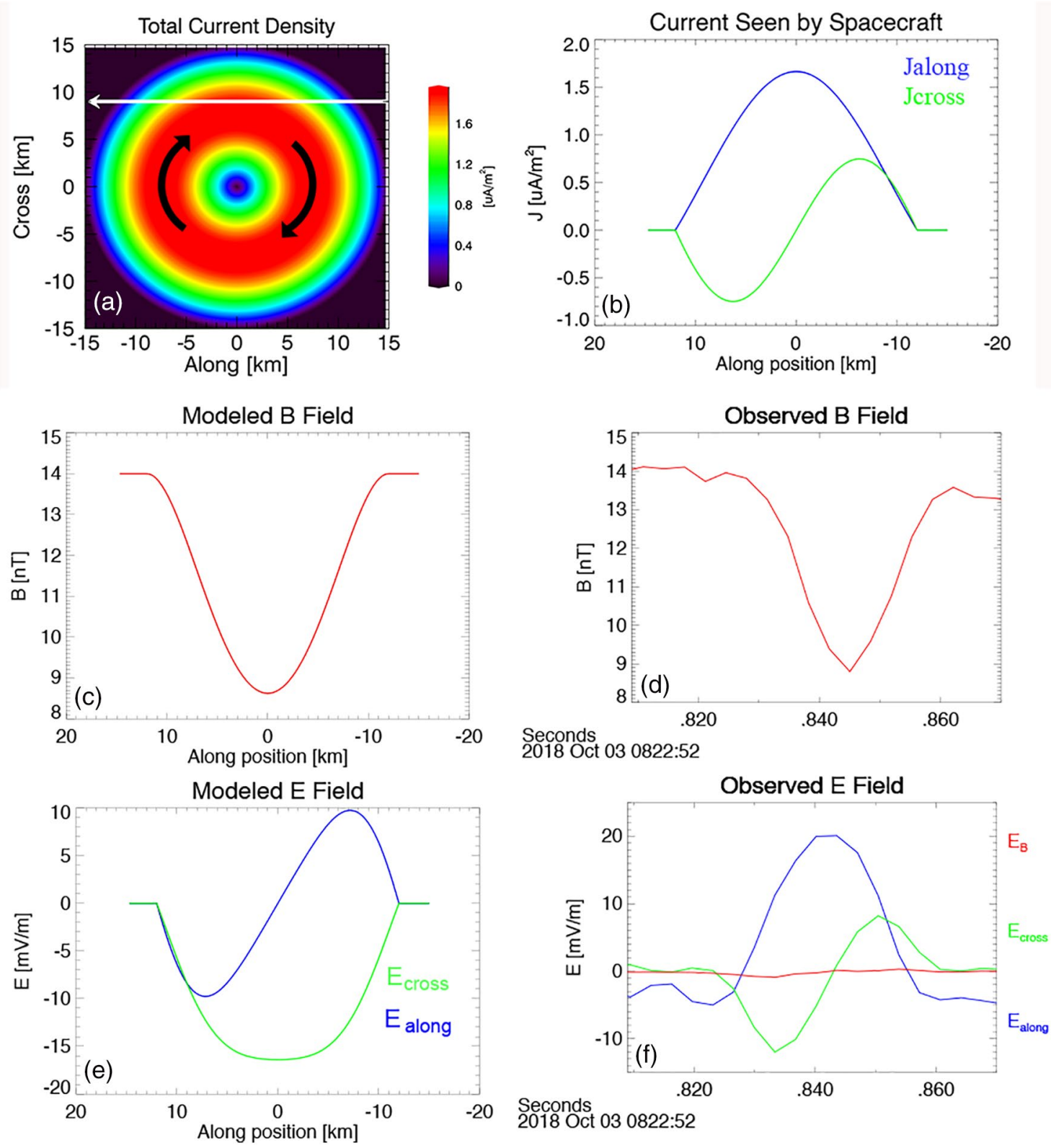

Figure 3. (a) Two-dimensional view of total current density $|\mathbf{J}|$ of an electron vortex as a function of spatial scale ( $X$ and $Y$ where the center of the vortex is $X=Y=0$ ), with a radius of $15 \mathrm{~km}$. The current density profile is defined in Equation 1 . The white arrow shows the spacecraft path across the structure. (b) The current density theoretically seen in both the $X$ and $Y$ directions along the given spacecraft path. The magnetic induced from the model is shown in (c) and compared to observations (d) from Parker in the Venusian magnetosheath. The electric field derived from the model (e) is also compared to observations (f).

instruments. Another source may be an instrumental effect that is, currently not well understood by the Parker community. All of the above may influence our analysis.

\section{Discussion}

In the previous section, we constructed an electron current vortex model with the intention of recreating observations from the Parker Solar Probe in the Venusian magnetosheath. This model is consistent with most of the characteristics of observed subproton-scale magnetic holes. The current vortex model matches the estimated size of the observed magnetic hole. The induced magnetic field from the model is also consistent (within $2 \%$ ) with the $\Delta|\mathbf{B}|$ observed by PSP. The model also produced electric fields with amplitudes 
similar to those observed (on the order of $10 \mathrm{mV} / \mathrm{m}$, within 35\%). The electric fields induced in the model, however, does not match the orientation of the fields seen in the observations. In fact, the observed electric fields deviate $\sim 90^{\circ}$ from the model.

The electric fields from all other magnetic hole candidates were also rotated in the plasma frame. All candidates deviated close to $90^{\circ}$ in the azimuthal direction from the model, in addition to the candidate in Figure 3. This suggests the deviation is related to a systematic or instrumental issue, rather than an issue from the plasma itself.

The electric fields were rotated according to proton velocity measurements from SPC. Velocity moments from SPAN-ion were also examined, but also resulted in a $90^{\circ}$ deviation from the model. However, it is possible that, within the Venusian magnetosheath, the full plasma distribution was not measured. SPC is directed sunward and requires the core of the plasma distribution to be within $30^{\circ}$ of its field-of-view (FOV) before the measurement degrades. Due to the orientation of the spacecraft, SPAN-ion was not pointed in the ram flow direction for the VGA1. The consequence is that only a partial distribution function of ions was measured, which affects and partially skews the derived plasma parameters. Velocities moments will inherently contain this offset if the core of the distribution is not in the FOV. A general estimate suggests that a $\sim 495 \mathrm{~km} / \mathrm{s}$ velocity in the direction perpendicular to the magnetic field would be needed to alter the direction of the flow such that the electric field would match the model. This would translate to $(433,-25$, 237) $\mathrm{km} / \mathrm{s}$ in the spacecraft frame. Considering SPC has a $30^{\circ} \mathrm{FOV}$ centered in the $+Z$ direction, this could be considered reasonable in the Venusian magnetosheath. However, much more investigation regarding the performance of the PSP particle instruments at Venus must be performed before this can be confirmed.

Additionally, the $90^{\circ}$ deviation from the model may be revealing of unforeseen errors in either the measurement or calibration of the electric field instrument on Parker Solar Probe. It has been shown a rotational error may exist for lower frequency $(<10 \mathrm{~Hz}$ ) electric field measurements by Mozer et al. (2020). It is unclear at this time whether this error can extend to higher frequency signals like the ones examined in this study. Further investigation is needed to determine all possible instrumental sources of error. This, however, is beyond the scope of this work.

While the orientation of the observed electric field differs from those induced from the current vortex model by $90^{\circ}$, the spatial size, $\mathbf{E}$ field amplitude, and induced $\Delta|\mathbf{B}|$ of the model are remarkably consistent with all observations. While the orientation of the electric field highlights specialized analysis is necessary during VGA1, there is sufficient evidence to support that these magnetic hole signatures are consistent with electron current vortices.

According to our analysis, a current vortex with an amplitude of $1.75 \mu \mathrm{A} / \mathrm{m}^{2}$ is required to induce the observed decrease in $|\mathbf{B}|$ shown in Figures 2 and 3. The electric fields seen with these $|\mathbf{B}|$ decreases suggest the current corresponds to electrons traveling at speeds on the order of $1,000 \mathrm{~km} / \mathrm{s}$, up to 5 times faster than the observed proton velocity moments. Moreover, at least eleven subproton-scale magnetic holes were identified throughout PSP's encounter with Venus. This suggests these structures are a common structure within the Venusian magnetosheath.

As stated previously, subproton-scale magnetic holes have arisen in multiple plasma turbulence simulations (Haynes et al., 2015; Roytershteyn et al., 2015). They have been suggested as a coherent structure that can arise naturally through turbulence. Observations in the terrestrial magnetosheath have also shown that subproton-scale magnetic holes can be seen with electron trapping (Huang et al., 2017) and electron heating perpendicular to the magnetic field (H. Liu et al., 2019). It is therefore possible that these structures may play a role or be a signature of turbulent dissipation. It is also possible they have evolved from other mechanisms (e.g., the mirror or tearing instability). What is clear, however, is the process that generates subproton-scale magnetic holes are present at both Earth and Venus.

\section{Conclusion}

On October 3, 2018, the Parker Solar Probe spacecraft encountered the Venusian magnetosheath as part of a gravity assist maneuver. During this encounter, localized depressions in magnetic field strength were observed with spatial scales less than the local thermal proton gyroradius, consistent with characteristics of 
subproton-scale magnetic holes. Eleven subproton-scale magnetic hole candidates were identified within the Venusian magnetosheath. No candidates were found in the solar wind prior to the initial shock crossing.

Subproton-scale magnetic holes have been observed in many regions of the terrestrial magnetosphere with diverse plasma conditions. It is now clear, by additional reports of their presence at Venus, that they are indicative of a universal plasma process. Additionally, these observations, as well as the modeled comparison, suggest that the Venusian magnetosheath is host to widespread, large-amplitude, small-scale, electron current structures. It is unclear how such structures manifest or how they affect their plasma environment. Their importance to Venusian microphysics is consequently unclear. Understanding them, however, can lead to unprecedented insights to the microphysical processes that occur within the Venusian magnetosphere.

The Parker Solar Probe mission will engage in a total of seven flybys of Venus. These flybys cover multiple regions of the Venusian space plasma environment, including the bow shock, foreshock, and magnetotail. With the advanced capabilities available on Parker Solar Probe, we stand to gain a better understanding of the microphysics that take place at Venus than we ever had and place those processes within the broader context of planetary electrodynamics across the inner solar system.

\section{Data Availability Statement}

The data is available via http://fields.ssl.berkeley.edu/.

\section{References}

Parker Solar Probe was designed, built, and is now operated by the Johns Hopkins Applied Physics Laboratory as part of NASA's Living with a Star (LWS) program (contract NNN06AA01C). Support from the LWS management and technical team has played a critical role in the success of the Parker Solar Probe mission. 24(12), 122121. https://doi.org/10.1063/1.5003017
Ahmadi, N., Germaschewski, K., \& Raeder, J. (2017). Simulation of magnetic holes formation in the magnetosheath. Physics of Plasmas,

Bale, S. D., Goetz, K., Harvey, P. R., Turin, P., Bonnell, J. W., de Wit, T., et al. (2016). The FIELDS Instrument Suite for Solar Probe Plus. Space Science Reviews, 204(1), 49-82. https://doi.org/10.1007/s11214-016-0244-5

Balikhin, M. A., Pokhotelov, O. A., Walker, S. N., Boynton, R. J., \& Beloff, N. (2010). Mirror mode peaks: THEMIS observations versus theories. Geophysical Research Letters, 37(5), L05104. https://doi.org/10.1029/2009gl042090

Balikhin, M. A., Sibeck, D. G., Runov, A., \& Walker, S. N. (2012). Magnetic holes in the vicinity of dipolarization fronts: Mirror or tearing structures? Journal of Geophysical Research: Space Physics, 117(8), 1-14. https://doi.org/10.1029/2012JA017552

Califano, F., Hellinger, P., Kuznetsov, E., Passot, T., Sulem, P. L., \& Trávníček, P. M. (2008). Nonlinear mirror mode dynamics: Simulations and modeling. Journal of Geophysical Research, 113(A8), A08219. https://doi.org/10.1029/2007JA012898

Case, A. W., Kasper, J. C., Stevens, M. L., Korreck, K. E., Paulson, K., Daigneau, P., et al. (2020). The Solar Probe Cup on the Parker Solar Probe. The Astrophysical Journal Supplement Series, 246(2), 43. https://doi.org/10.3847/1538-4365/ab5a7b

Fox, N. J., Velli, M. C., Bale, S. D., Decker, R., Driesman, A., Howard, R. A., et al. (2016). The Solar Probe Plus Mission: Humanity's First Visit to Our Star. Space Science Reviews, 204(1), 7-48. https://doi.org/10.1007/s11214-015-0211-6

Ge, Y. S., McFadden, J. P., Raeder, J., Angelopoulos, V., Larson, D., \& Constantinescu, O. D. (2011). Case studies of mirror-mode structures observed by THEMIS in the near-Earth tail during substorms. Journal of Geophysical Research, 116(A1), A01209. https://doi. org/10.1029/2010JA015546

Gershman, D. J., Dorelli, J. C., Viñas, A. F., Avanov, L. A., Gliese, U., Barrie, A. C., et al. (2016). Electron dynamics in a subproton-gyroscale magnetic hole. Geophysical Research Letters, 43(9), 4112-4118. https://doi.org/10.1002/2016GL068545

Goodrich, K. A., Ergun, R. E., \& Stawarz, J. E. (2016). Electric fields associated with small-scale magnetic holes in the plasma sheet: Evidence for electron currents. Geophysical Research Letters, 43(12), 6044-6050. https://doi.org/10.1002/2016GL069601

Goodrich, K. A., Ergun, R. E., Wilder, F. D., Burch, J., Torbert, R., Khotyaintsev, Y., et al. (2016). MMS Multipoint electric field observations of small-scale magnetic holes. Geophysical Research Letters, 43(12), 5953-5959. https://doi.org/10.1002/2016GL069157

Halekas, J. S., Whittlesey, P., Larson, D. E., McGinnis, D., Maksimovic, M., Berthomier, M., et al. (2020). Electrons in the Young Solar Wind: First Results from the Parker Solar Probe. The Astrophysical Journal Supplement Series, 246(2), 22. https://doi.org/10.3847/1538-4365/ ab4cec

Hasegawa, A. (1969). Drift mirror instability in the magnetosphere. Physics of Fluids, 12(12), 2642-2650. https://doi.org/10.1063/1.1692407

Haynes, C. T., Burgess, D., Camporeale, E., \& Sundberg, T. (2015). Electron vortex magnetic holes: A nonlinear coherent plasma structure. Physics of Plasmas, 22(1), 012309. https://doi.org/10.1063/1.4906356

Huang, S. Y., Sahraoui, F., Yuan, Z. G., He, J. S., Zhao, J. S., Contel, O. L., et al. (2017). Magnetospheric Multiscale Observations of Electron Vortex Magnetic Hole in the Turbulent Magnetosheath Plasma. The Astrophysical Journal, 836(2), L27. https://doi. org/10.3847/2041-8213/aa5f50

Ji, X.-F., Wang, X.-G., Sun, W.-J., Xiao, C.-J., Shi, Q.-Q., Liu, J., \& Pu, Z.-Y. (2014). EMHD theory and observations of electron solitary waves in magnetotail plasmas. Journal of Geophysical Research: Space Physics, 119(6), 4281-4289. https://doi.org/10.1002/2014JA019924

Johnson, J. R., \& Cheng, C. Z. (1997). Global structure of mirror modes in the magnetosheath. Journal of Geophysical Research, 102(A4), 7179-7190. https://doi.org/10.1029/96JA03949

Kasper, J. C., Abiad, R., Austin, G., Balat-Pichelin, M., Bale, S. D., Belcher, J. W., et al. (2016). Solar Wind Electrons Alphas and Protons (SWEAP) Investigation: Design of the Solar Wind and Coronal Plasma Instrument Suite for Solar Probe Plus. Space Science Reviews, 204(1), 131-186. https://doi.org/10.1007/s11214-015-0206-3

Kuznetsov, E. A., Passot, T., \& Sulem, P. L. (2008). Nonlinear theory of mirror instability near its threshold. JETP Letters, 86(10), 637-642. https://doi.org/10.1134/S0021364007220055 
Li, Z. Y., Sun, W. J., Wang, X. G., Shi, Q. Q., Xiao, C. J., Pu, Z. Y., et al. (2016). An EMHD soliton model for small-scale magnetic holes in magnetospheric plasmas. Journal of Geophysical Research: Space Physics, 121(5), 4180-4190. https://doi.org/10.1002/2016JA022424

Liu, H., Zong, Q. G., Zhang, H., Xiao, C. J., Shi, Q. Q., Yao, S. T., et al. (2019). MMS observations of electron scale magnetic cavity embedded in proton scale magnetic cavity. Nature Communications, 10(1), 1-11. https://doi.org/10.1038/s41467-019-08971-y

Liu, J., Yao, S. T., Shi, Q. Q., Wang, X. G., Zong, Q. G., Feng, Y. Y., et al. (2020). Electron Energization and Energy Dissipation in Microscale Electromagnetic Environments. The Astrophysical Journal Letters, 899(2), L31. https://doi.org/10.3847/2041-8213/abab92

Liu, Y. Y., Fu, H. S., Zong, Q. G., Wang, Z., Liu, C. M., Huang, S. Y., et al. (2020). First Topology of Electron-Scale Magnetic Hole. Geophysical Research Letters, 47(18), 1-9. https://doi.org/10.1029/2020GL088374

Malaspina, D. M., Ergun, R. E., Bolton, M., Kien, M., Summers, D., Stevens, K., et al. (2016). The Digital Fields Board for the FIELDS instrument suite on the Solar Probe Plus mission: Analog and digital signal processing. Journal of Geophysical Research: Space Physics, 121(6), 5086-5096. https://doi.org/10.1002/2016JA022344

Mozer, F. S., Agapitov, O. V., Bale, S. D., Bonnell, J. W., Bowen, T. A., \& Vasko, I. (2020). DC and Low-Frequency Electric Field Measurements on the Parker Solar Probe. Journal of Geophysical Research: Space Physics, 125(9), e2020JA027980. https://doi.org/10.1029/2020JA027980

Roytershteyn, V., Karimabadi, H., \& Roberts, A. (2015). Generation of magnetic holes in fully kinetic simulations of collisionless turbulence. Philosophical Transactions of the Royal Society A: Mathematical, Physical and Engineering Sciences, 373(2041), 20140151. https:// doi.org/10.1098/rsta.2014.0151

Russell, C. T., Jian, L. K., Luhmann, J. G., Zhang, T. L., Neubauer, F. M., Skoug, R. M., et al. (2008). Mirror mode waves: Messengers from the coronal heating region. Geophysical Research Letters, 35(15), L15101. https://doi.org/10.1029/2008GL034096

Soucek, J., Lucek, E., \& Dandouras, I. (2008). Properties of magnetosheath mirror modes observed by Cluster and their response to changes in plasma parameters. Journal of Geophysical Research, 113(A4), A04203. https://doi.org/10.1029/2007JA012649

Southwood, D. J., \& Kivelson, M. G. (1993). Mirror instability: 1. Physical mechanism of linear instability. Journal of Geophysical Research, 98(A6), 9181-9187. https://doi.org/10.1029/92ja02837

Stix, T. H. (1992). Waves in plasmas. AIP-Press.

Sun, W. J., Shi, Q. Q., Fu, S. Y., Pu, Z. Y., Dunlop, M. W., Walsh, A. P., et al. (2012). Cluster and TC-1 observation of magnetic holes in the plasma sheet. Annales Geophysicae, 30(3), 583-595. https://doi.org/10.5194/angeo-30-583-2012

Sundberg, T., Burgess, D., \& Haynes, C. T. (2015). Properties and origin of subproton-scale magnetic holes in the terrestrial plasma sheet. Journal of Geophysical Research: Space Physics, 120(4), 2600-2615. https://doi.org/10.1002/2014JA020856

Tenerani, A., Califano, F., Pegoraro, F., \& Le Contel, O. (2012). Coupling between whistler waves and slow-mode solitary waves. Physics of Plasmas, 19(5), 52103. https://doi.org/10.1063/1.4717764

Tenerani, A., Contel, O. L., Califano, F., Robert, P., Fontaine, D., Cornilleau-Wehrlin, N., \& Sauvaud, J.-A. (2013). Cluster observations of whistler waves correlated with ion-scale magnetic structures during the 17 August 2003 substorm event. Journal of Geophysical Research: Space Physics, 118(10), 6072-6089. https://doi.org/10.1002/jgra.50562

Whittlesey, P. L., Larson, D. E., Kasper, J. C., Halekas, J., Abatcha, M., Abiad, R., et al. (2020). The Solar Probe ANalyzers-Electrons on the Parker Solar Probe. The Astrophysical Journal Supplement Series, 246(2), 74. https://doi.org/10.3847/1538-4365/ab7370

Wintertialter, D., Neugebauer, M., Goldstein, B. E., Smith, E. J., Bame, S. J., \& Balogh, A. (1994). Ulysses field and plasma observations of magnetic holes in the solar wind and their relation to mirror-mode structures. Journal of Geophysical Research, 99(A12), 23371-23381. https://doi.org/10.1029/94JA01977

Yao, S. T., Wang, X. G., Shi, Q. Q., Pitkänen, T., Hamrin, M., Yao, Z. H., et al. (2017). Observations of kinetic-size magnetic holes in the magnetosheath. Journal of Geophysical Research: Space Physics, 122(2), 1990-2000. https://doi.org/10.1002/2016JA023858 